Research Paper

\title{
Distributed Features of Vimentin-Containing Neural Precursor Cells in Olfactory Bulb of SOD1G93A Transgenic Mice: a Study about Resource of Endogenous Neural Stem Cells
}

\author{
Chunyan Tang ${ }^{1 *}$, Lei Zhu1 ${ }^{*}$, Weiming Gan ${ }^{1 *}$, Huiting Liang ${ }^{1 *}$, Jiao Li $^{1^{*}}$, Jie Zhang ${ }^{*}$, Xiong Zhang ${ }^{2,3^{*}}$, Yi Lu, \\ Renshi $\mathrm{Xu}^{1 凶}$ \\ 1. Department of Neurology, the First Affiliated Hospital of Nanchang University, Nanchang 330006, Jiangxi, China; \\ 2. Graduate School of Southern Medical University, Guangzhou 510515, Guangdong Province, China; \\ 3. Department of Neurology, Guangdong General Hospital, Guangdong Academy of Medical Sciences, Guangdong Neuroscience Institute, Guangzhou \\ 510080, Guangdong Province, China. \\ *The authors equally contributed to the work, was the jointed first authors.
}

$\square$ Corresponding author: Prof. Renshi Xu. Department of Neurology, the First Affiliated Hospital of Nanchang University, Nanchang 330006, Jiangxi, P.R. China. Email address: xurenshi@ncu.edu.cn. Telephone number: +86+791+88603798.

( ) Ivyspring International Publisher. Reproduction is permitted for personal, noncommercial use, provided that the article is in whole, unmodified, and properly cited. See http://ivyspring.com/terms for terms and conditions.

Received: 2016.07.02; Accepted: 2016.09.01; Published: 2016.10.26

\begin{abstract}
No any effective treatments can prevent from the motor neuron degeneration in amyotrophic lateral sclerosis (ALS) at present. In order to modulating the endogenous neural precursor cells (NPCs) to repairing the degenerative motor neurons in ALS, we studied the alteration of endogenous vimentin-containing NPCs in olfactory bulb (OB) at the different stages of SOD1 wlid-type and G93A transgenic mice. The results showed that the vimentin-containing cells (VCCs) were mainly distributed in the glomerular layer $(\mathrm{Gl})$, the accessory $O B(A O B)$, the $O B$ core, the granular cell layer (GRO) and the mitral cell layer (MI)+the internal plexiform layer (IPL) of the OB of adult mice. Almost all VCCs in $\mathrm{GI}, \mathrm{OB}$ core and GRO were the GFAP positive cells. Almost all VCCs in AOB were the Oligo-2 positive cells. Fewer VCCs in MI+IPL were the NeuN positive cells. VCCs significantly increased in the OB core and $\mathrm{Gl}$ of adult $\mathrm{OB}$ at the pre-onset, onset and progression stages of ALS-like G93A transgenic disease, particularly in OB core. All increased VCCs were the GFAP positive cells. Our data suggested that there extensively existed the endogenous vimentin-containing NPCs in the OB of adult mice, which was a potential resource of neural stem cells, they could differentiate into astrocyte, oligodendrocyte and neuron cells, were a potential astrocyte neuroregenerative response in adult OB in the ALS-like disease, were a potential pathway to repair the degenerated motor neurons.
\end{abstract}

Key words: Amyotrophic lateral sclerosis, Olfactory bulb, Vimentin, Neural precursor cells, Astrocyte cell, Neural stem cells, SOD1 G93A transgenic mouse.

\section{Introduction}

Amyotrophic lateral sclerosis (ALS) is a relentlessly progressive neurodegenerative disorder, the prevalence rate is up to $7.4 / 100,000$, the overall risk of ALS development in a lifetime is 1:400. ALS is characterized by the degeneration of motor neurons in the cerebral motor cortex, medulla and spinal cord, which results in the progressive muscle paralysis, ultimately dies of the respiratory failure [1-4]. To date, only one pharmaceutical agent called as riluzole has been shown to afford a benefit on the survival time of ALS patients [5-7]. Some agents have been identified to have the effect on the treatment of ALS in the preclinical models of ALS, but which subsequently were not found to be efficacy to the ALS patients in the clinical trials $[8,9]$. Therefore, currently, no any treatments can prevent from the progression of ALS. 
The transplantation of the neural stem cells (NST) takes it for granted to be an ideal cured method of ALS, which was sought after by many researchers. However, still to now, it has not obtained the satisfactory results [10-14].

Early efforts to transplant stem cells mainly focused on the motor neuron replacement, but has not obtained the ideal effects up to now [15-17]. Some studied results of the animal and tissue culture models suggested that despite ALS was a disease of relatively selective motor neurons death, the non-neuronal cells also were found to be closely associated with the neuronal dysfunction and death [18-20]. Therefore, the recent studies have transferred to focus on the transplantation of the mesenchymal stem cells, or the glial progenitors in the transgenic mouse or rat models of ALS, the results revealed that the transplantation of non-neuronal cells exerts the neuron protection through a variety of different mechanisms including the neurotrophic factor secretion, the regulation of glutamate transporter, the modulation of neuron inflammation and others [10-14, 21-25].

Although the exogenous replacement of neural cells and not neural cells can produce some biological effects, they exist a lot of hard overcome deficiencies. For example, it almost isn't possible that the exogenous transplantation of stem cells generates the sustained proliferation and differentiation, migrates to the pathologically damaged regions, and promotes the functional reconstruction of neural structures, as well as exerts the same physiological function of the normal neural cells at the physiological condition [16, 25-30]. In addition, there are a series of side effects caused by the transplantation of the exogenous stem cells, such as the immunological rejection responses, the local tumor proliferation, the insufficient resources of exogenous transplantated cells, the neural injury of transplantation operation and so on [31-33]. These biologically insufficient factors and the side effects of transplantation seriously hampered the transplantation development of the exogenous stem cells in the therapy of ALS.

Therefore, it is necessary to further search the other ways of ALS treatment. In our previous studies, we found that the endogenous neural precursor cells (NPCs) could proliferate, differentiate and migrate to the damaged regions of spinal cord, produce the novel neural cells including neuron and glial cells, partially repair the neuron lesion in the SOD1 G93A transgenic mice, but the repair ability was very less, could not effectively ameliorate or prevent the disease development $[10,11,34]$. In view of this, we speculated that if the mechanism of the proliferation, differentiation and migration of endogenous NPCs in the pathological conditions of ALS could be elucidated, it would bring the possibility to us to regulate the proliferation, differentiation and migration of endogenous NPCs, promote the self-recovering ability, enhance its neural repair function, improve the self-repair ability of the neural functional lesions in ALS, ameliorate or prevent from the development of ALS, and also could avoid the deficiency of the exogenous stem cell transplantation, would be a potential novel ideal way to ameliorate or prevent from the development of ALS besides the transplantation of exogenous stem cell. Therefore, to clarify the distributed features of NPCs in the disease courses of ALS is one of key steps further investigating the regulation of NPCs.

Our previous study found that the olfactory bulb (OB) in mice was one of anatomic regions where NPCs abundantly distributed [35], therefore, in this study, we chose to systematically observe and analyze the distributed features of vimentin-containing NPCs in $\mathrm{OB}$, including its proliferation, differentiation and migration in the different anatomic regions and cell types of $\mathrm{OB}$ at the pre-onset, onset and progression stages of the SOD1 wild-type and G93A transgenic mice, it aimed to search the possible changed mechanisms of NPCs in the pathogenic condition of ALS and find the target modulating the NPCs. Our experimental results revealed that there existed an extensive endogenous vimentin-containing NPCs distribution in the $\mathrm{OB}$ of normal adult mice, particularly in the glomerular layer $(\mathrm{Gl})$, the accessory olfactory bulb (AOB), the olfactory bulb core (OB core), the granular cell layer (GRO) and the mitral cell layer (MI)+the internal plexiform layer (IPL), they could differentiate into the astrocyte, oligodendrocyte glial cells and the neuron cells, these NPCs were a potential neuroregenerative resources in the CNS of normal adult mice. NPCs significantly increased in the $\mathrm{OB}$ core and the $\mathrm{Gl}$ of adult $\mathrm{OB}$ at the pre-onset, onset and progression stages, all increase NPCs differentiated into the astrocyte cell in the ALS-like SOD1 G93A transgenic mice, which indicated an astrocyte neuroregenerative response from the vimentin-containing NPCs in the $\mathrm{OB}$ of the SOD1G93A transgenic mice. Our data about the distributed features of NPCs in the OB of ALS-like SOD1 G93A transgenic mice provide some novel evidences for further studying the regulation of NPCs in the treatment of ALS.

\section{Materials and methods}

\section{Study approval}

The animal study was conducted in accordance with the Guide for the Care and Use of Laboratory 
Animals, and was reviewed and approved by the ethics committee for animal care and use of the First Affiliated Hospital of Nanchang University, China.

\section{Animals}

The line of the C57BL/6 SOD1 G93A mice from Jackson laboratory, Bar Harbour, Maine was maintained by mating the SOD1 G93A transgenic male mice with the wild-type female mice in the neurological laboratory of the First Affiliated Hospital of Nanchang University. The SOD1 G93A transgenic mice were detected whether or not the positive SOD1 G93A transgenic mice by the PCR of the genomic DNA derived from the tail of mice. The following were the used primers: The reward-primer of hmSOD1 G93A was 5'-CAT CAG CCC TAA TCC ATC TGA-3', the reverse-primer of hmSOD1 G93A was 5'-CGC GAC TAA CAA TCA AAG TGA-3', the forward-primer of IL-2 was 5'-CTA GGC CAC AGA ATT GAA AGA TCT-3', the reverse-primer of IL-2 was 5'-GTA GGT GGA AAT TCT AGC ATC ATC C-3'. The conditions of PCR amplification were: $94^{\circ} \mathrm{C}$ degeneration for 3 seconds, $60^{\circ} \mathrm{C}$ annealing for 1 minutes and $72^{\circ} \mathrm{C}$ extension for 1 minute, total cycles were 35 times. The experimental animals were sacrificed at the time points of pre-onset $(60-70 \mathrm{~d})$, onset (90-100 d) and progressive (120-130 d) stages of SOD1 G93A transgenic mice and the age-matched wild-type mice. At the different stages of disease, the gastrocnemius muscles of the abnormal limb were performed the HE stain, observed the alterations of muscle structure in the light microscope, further confirmed the severity of muscle atrophy in the paralysis limb and distinguished the disease stages of pre-onset, onset and progression [34, 36-38]. The protocol of muscle biopsy was the same as the descriptions in our previously published paper [34].

\section{Fluorescent immunohistochemical stain of $O B$}

The SOD1 wild-type and G93A transgenic mice were anesthetized and perfused using $20 \mathrm{ml}$ of $0.9 \%$ saline and $40 \mathrm{ml}$ of $4 \%$ paraformaldehyde in $1 \times$ PBS $(\mathrm{pH} 7.5)$ at room temperature. $\mathrm{OB}$ was excised and placed in $4 \%$ paraformaldehyde buffer overnight, incubated in $20 \%$ sucrose in 1xPBS (pH 7.5), embedded using optimum cutting temperature $(O C T)$. OB was coronally and successively cut into 12 $\mu \mathrm{m}$ sections from the rostral to the caudal on a Leica cryostat and collected on the Superfrost Plus slides. In the fluorescent immunohistochemical stain, the sections of $\mathrm{OB}$ were permeabilized using $0.2 \%$ TritonX-100 and blocked using $10 \%$ goat serum in 1xPBS after rehydrated in 1xPBS (pH 7.4), incubated using the following antibodies: Vimentin 1:100 (Santa cruz biotechnology Inc); NeuN 1:250, GFAP 1:1000 and Oligo-2 1:100 (Abcam, Hong Kong Ltd.) at $4{ }^{\circ} \mathrm{C}$ overnight, followed by washing 6 times with $0.2 \%$ Triton X-100 in 1xPBS, incubating using the secondary antibodies (Donkey anti goat, 1:250, donkey anti rabbit, 1:200) conjugated to fluorescence (Green) or/and rhodamine (Red) for 2 hours at room temperature, and DAPI stained (Blue), extensively washing for 5 times, each for 5 minutes, mounting using antifade medium, examining in a Nikon E800 fluorescent microscope equipped with a spot digital camera (Diagnostic Instruments, Sterling Heights, MI, USA) and Photoshop software (Adobe Systems, San Jose, CA, USA), and taking pictures. Multiple labeled histochemical stain conjugated to anti-Vimentin, NeuN, GFAP, Oligo-2 antibodies and DAPI was used to observe and analyze the cell proliferation, migration and the cell type of differentiation.

\section{Analysis of fluorescent immunohistochemical positive cells}

The analysis of the fluorescent immunohistochemical positive cells was performed by the following steps: 1). Counted the amount of positive cells in the distinct regions of $\mathrm{OB}$ at 200 magnifications in 10 successive slices of the similar anatomic structure, 3 sections each slice and 3 mice per group were used; 2). Calculated the total sum of positive cells of all sections, the total sum was divided by the total number of sections, the averaged amount was used for the quantitative analysis.

\section{Statistical analysis}

The experimental data were expressed using mean $\pm \mathrm{SD}$. The specific comparison between the control and the experimental individual was analyzed using Student's t-tests, $\mathrm{P}<0.05$ was considered as the statistically significant difference.

\section{Results}

\section{The identification of C57BL/6J SODI G93A transgenic mice}

We screened and determined the C57BL/6 J SOD1 G93A positive transgenic mice by the PCR of genomic DNA and the muscle biopsy which were the same as our previous published paper [34]. In our breeding SOD1 G93A transgenic mice, we observed the typical characteristics of ALS-like symptoms at the pre-onset (60-70 d), onset (90-100 d) and progression (120-130 d) stages in the ALS-like disease courses. The SOD1 G93A transgenic mice usually suffered from the strong gait impairment, the muscle atrophy and the reduced motor function. 
The distributed features of vimentincontaining cells (VCCs) in the adult OB of the SOD 1 wild-type mice

The VCCs mainly distributed in the glomerular layer (Gl), the accessory olfactory bulb (AOB), the olfactory bulb core (OB core), the granular cell layer (GRO), the mitral cell layer (MI) and the internal plexiform layer (IPL) of the OB of SOD1 wild-type adult mice (Normal adult mice), a few of VCCs sparsely distributed in the other regions of OB. The VCCs distribution in the different anatomic regions of OB was significantly different, the rank order of total VCCs amount in the entire OB was Gl greater than $A O B$ greater than $O B$ core greater than $G R O$ greater than $\mathrm{MI}+\mathrm{IPL}$ greater than the others (i.e. $\mathrm{Gl}>\mathrm{AOB}>\mathrm{OB}$ core $>$ GRO $>$ MI+IPL $>$ the others) (Fig. 1). Total VCCs amount in the other regions of the entire OB wasn't significantly different. Total VCCs amount in the entire OB wasn't significantly different in the life-span time between 60 and 130 days (Fig. 2). The VCCs distribution in the different anatomic regions of $\mathrm{OB}$ didn't significantly change at the different life-span time between 60 and 130 days yet, the rank order of VCCs amount in the different anatomic regions of $\mathrm{OB}$ was $\mathrm{Gl}>\mathrm{AOB}>\mathrm{OB}$ core $>\mathrm{GRO}>\mathrm{MI}+\mathrm{IPL}>$ the others (Fig. 3). The rank order of VCCs density in the entire $\mathrm{OB}$ was $\mathrm{AOB}>\mathrm{OB}$ core $>\mathrm{GRO}>\mathrm{Gl}>\mathrm{MI}>$ the others (Fig. 4). Almost all VCCs in the Gl, OB core and GRO were the GFAP (Astrocyte marker) positive cells, which indicted that these VCCs were the astrocyte cells. Almost all VCCs in the AOB were the Oligo-2 (Oligodendrocyte marker) positive cells, which indicated that these VCCs were the oligodendrocyte cells. The fewer VCCs in the MI and IPL were the NeuN (Neuronal marker) positive cells, which indicated that these VCCs were the neuron cells (Fig. 4).

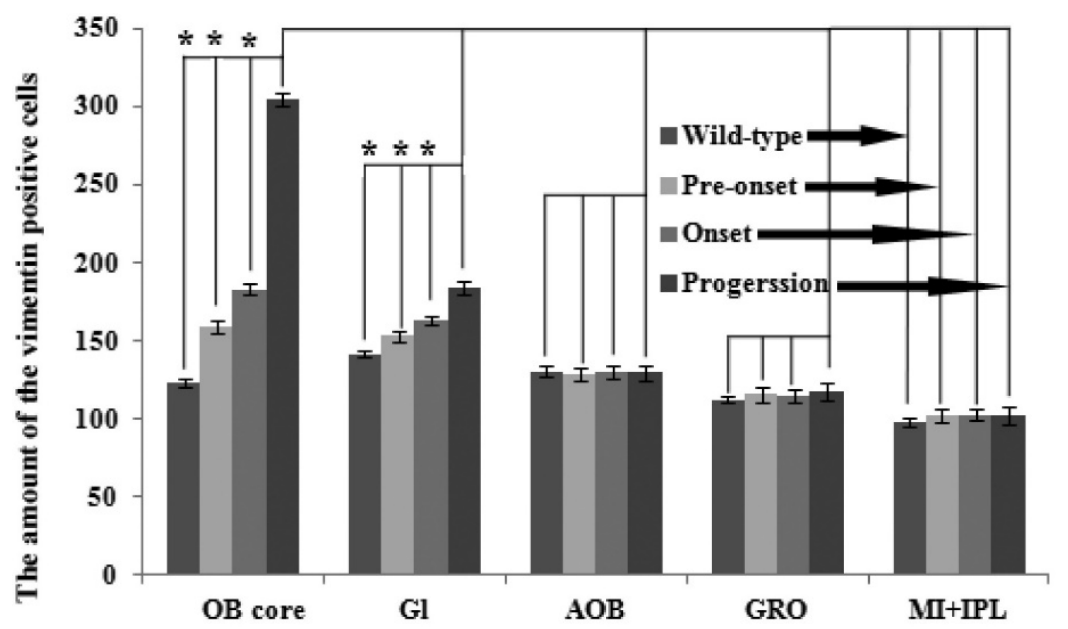

Figure 3. The comparison of vimentin positive cells amount in the different regions of $O B$ at the different stages of the SOD1 wild-type and G93A transgenic mice. The VCCs significantly increased in the $O B$ core and $\mathrm{Gl}$ of the adult $\mathrm{OB}$ at the pre-onset, onset and progression stages. Notably, there was a more significant increase of VCCs amount in the $\mathrm{OB}$ core during the pre-onset, onset and progression stages of the ALS-like SOD1 G93A transgenic mice compared to the age-matched wild-type mice.

The different regions of olfactory bulb in the different stages of adult SOD1 wild-type and G93A transgenic mice 


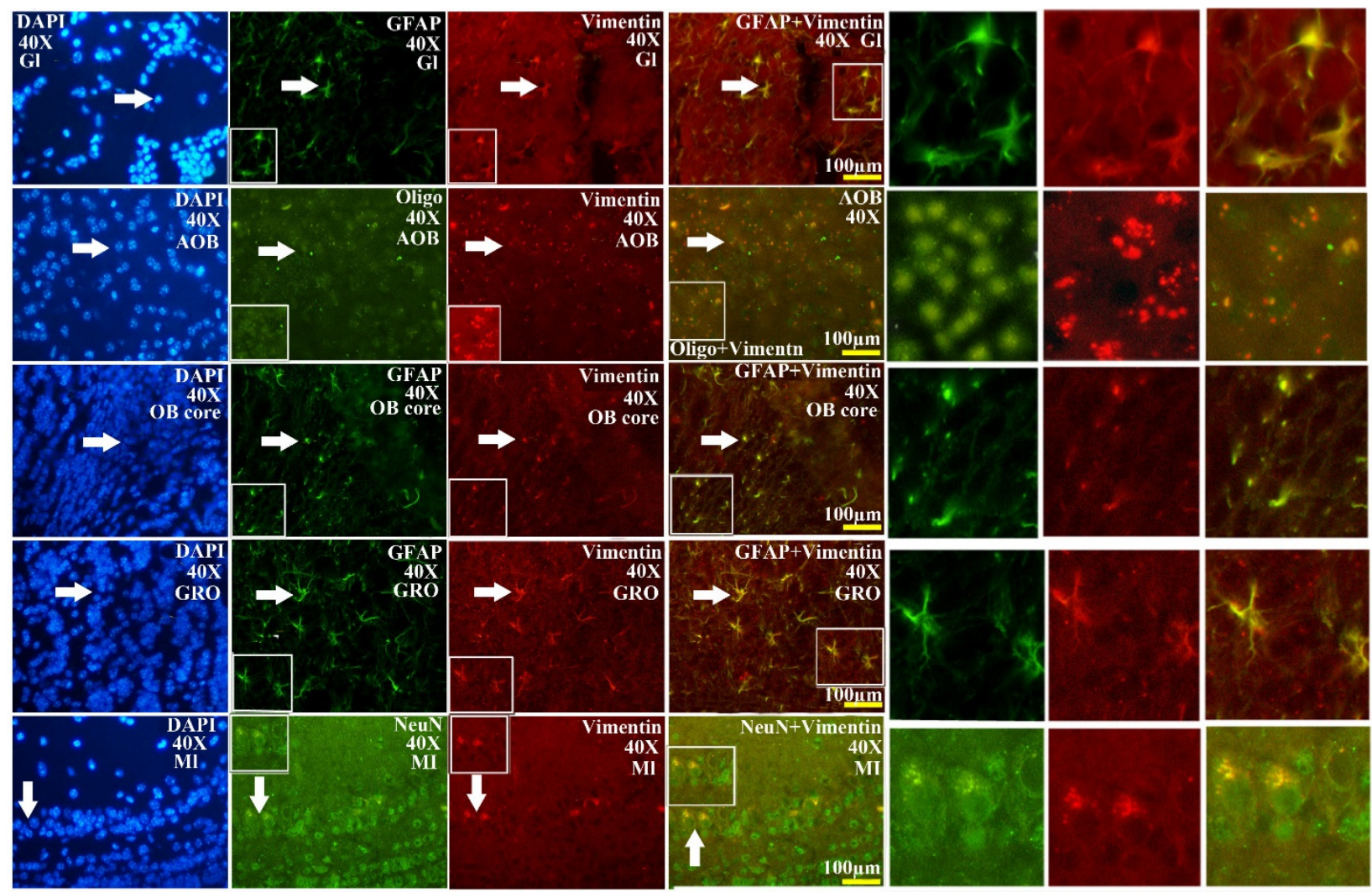

Figure 4. The representative images amplified by 40 times of vimentin positive cells distribution in the different regions and neural cells of OB in the SODI wild-type mice. The rank order of $V C C$ s density in the entire $O B$ was $A O B>O B$ core $>G R O>G \mid>M I>$ the others. Almost all VCCs in the $G l, O B$ core and $G R O$ were the astrocyte cell. Almost all NPCs in the AOB were the oligodendrocyte cell. The fewer NPCs in MI and IPL (No image) were the neuron cells. The white arrow indicated the positive cell.

\section{Distributed features of VCCs in the adult OB of the SOD1 G93A transgenic mice}

The further analysis of VCCs amount in the different regions of $\mathrm{OB}$ during the different ALS-like disease stages in the SOD1 G93A transgenic mice demonstrated that the VCCs distribution of OB in the different anatomic regions at the different disease stages showed a significant alteration, the rank order of VCCs amount in the OB core and Gl regions of OB was progression>onset>pre-onset>wild-type, that in the AOB, GRO, MI, IPL didn't significantly change. The results revealed that the VCCs significantly increased in the $\mathrm{OB}$ core and the $\mathrm{Gl}$ of adult $\mathrm{OB}$ at the pre-onset, onset and progression stages of the ALS-like SOD1 G93A transgenic mice compared to the age-matched wild-type mice (Fig. 3, 5, 6). Notably, there was a more significant increase of VCCs amount in the $\mathrm{OB}$ core during the pre-onset, onset and progression stages (Fig. 3, 5, 6). The VCCs in the other anatomic regions of $\mathrm{OB}$ including $\mathrm{AOB}, \mathrm{GRO}, \mathrm{MI}$ and IPL weren't found a significant decrease, which indicated that the VCCs didn't migrate between the different anatomic regions of $\mathrm{OB}$, the increased VCCs was a proliferation response (Fig. 3). All increased
VCCs were the GFAP positive cells, which indicted that all increased VCCs were the astrocyte cells (Fig. 5, 6).

\section{Discussion}

Our study demonstrated three major findings: 1). The VCCs extensively distributed in the Gl, AOB, OB core, GRO, MI and IPL of the OB of normal adult mice, only a few of VCCs sparsely distributed in the other regions, these VCCs were an abundant resource of NPCs and a potential resource of endogenous NST; 2). Almost all VCCs in the Gl, OB core and GRO were the astrocyte cells, almost all VCCs in the AOB were the oligodendrocyte cells, and the fewer VCCs in MI and IPL were the neuron cells in the OB of normal adult mice, which indicated that these NPCs of VCCs could differentiate into the astrocyte, oligodendrocyte and neuron cells, were a potential endogenous neuroregenerative source; 3 ). A significant increase of VCCs occurred in the $\mathrm{OB}$ core and Gl of $\mathrm{OB}$ during the pre-onset $(60-70 \mathrm{~d})$, onset $(90-100 \mathrm{~d})$ and progression (120-130 d) stages of the ALS-like SOD1 G93A transgenic mice, particularly in the OB core at the pre-onset, onset and progression stages. The VCCs 
didn't migrate between the different anatomic regions of OB, all increased VCCs in OB during the pre-onset, onset and progression stages of the ALS-like SOD1 G93A transgenic mice were a proliferation response, and differentiated into the astrocyte cell, which suggested that the increased VCCs were the endogenous NPCs of the astrocyte cells, but not neuron, oligodendrocyte and microgliocyte cells, was a potential NST that might be modulated their proliferation, differentiation and migration in the pathogenic condition of ALS (Fig. 7). Thus, this study provides some novel evidences that the abundant NPCs existed in the abnormal adult OB, which were a potential resource of NST. It might be a hopeful pathway repairing the degenerated motor neurons and/or stopping the development of ALS to stimulate the endogenous NPCs proliferation of $\mathrm{OB}$ and differentiate into the astrocyte, oligodendrocyte, microgliocyte and neuron cells at the pathological processes of ALS.

\section{The distributed features of VCCs in the adult OB of the SODI wild-type mice: an abundant resource of NPCs and a potential resource of NST}

In order to performing a comparison on the alteration of VCCs distribution in the adult OB of the ALS-like SOD1 G93A transgenic mice, we firstly analyzed the alteration of VCCs distribution in the adult OB of the SOD1 wild-type mice (Control), the results showed that the VCCs distribution in the different anatomic regions of normal adult $\mathrm{OB}$ were significantly different. The vast majority of VCCs were distributed in the $\mathrm{Gl}, \mathrm{AOB}, \mathrm{OB}$ core, GRO, MI and IPL, there were a few of VCCs sparsely distributed in the other regions. The VCCs amount in
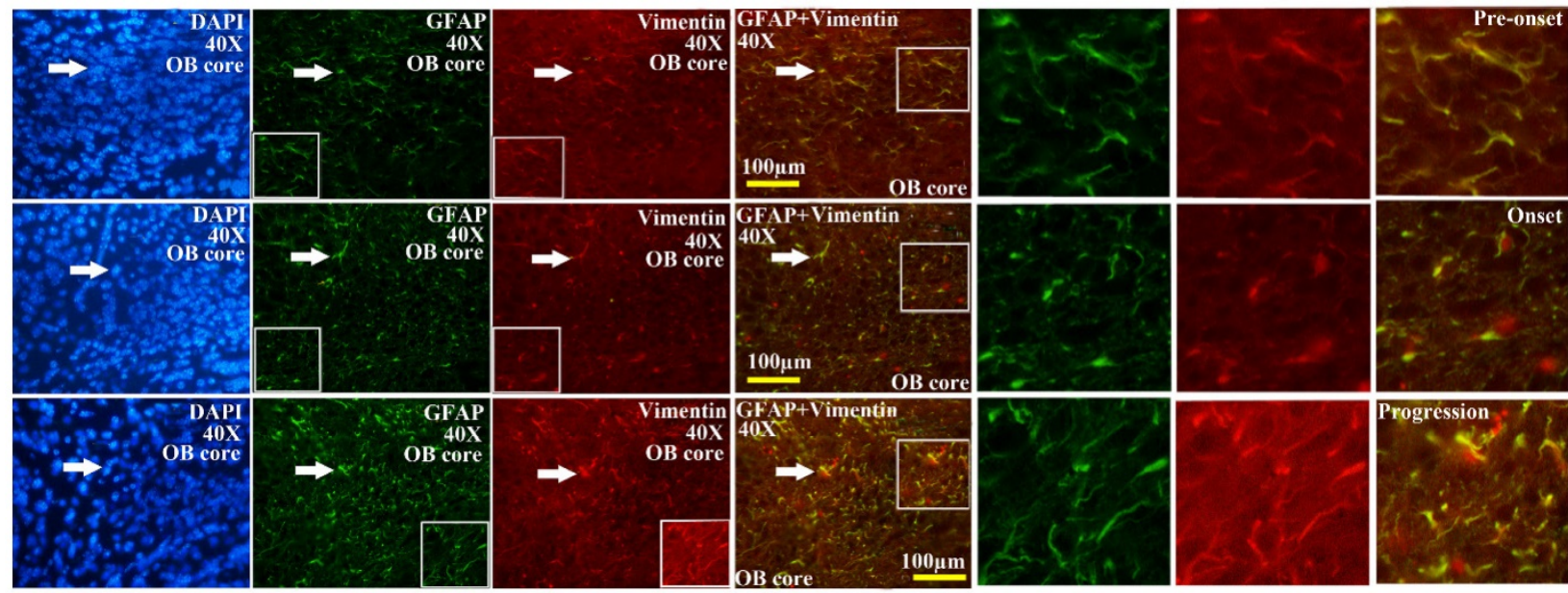

Figure 5. The representative images amplified by 40 times of vimentin positive cells distribution in the $O B$ core of $O B$ at the pre-onset, onset and progression stages of the SODI G93A transgenic mice. The amount of vimentin positive cells gradually increased from the pre-onset to onset to progression stages in the SODI G93A transgenic mice. The most significant increase stage of vimentin positive cells was at the progression stage. Almost increased vimentin positive cells were the astrocyte cells.
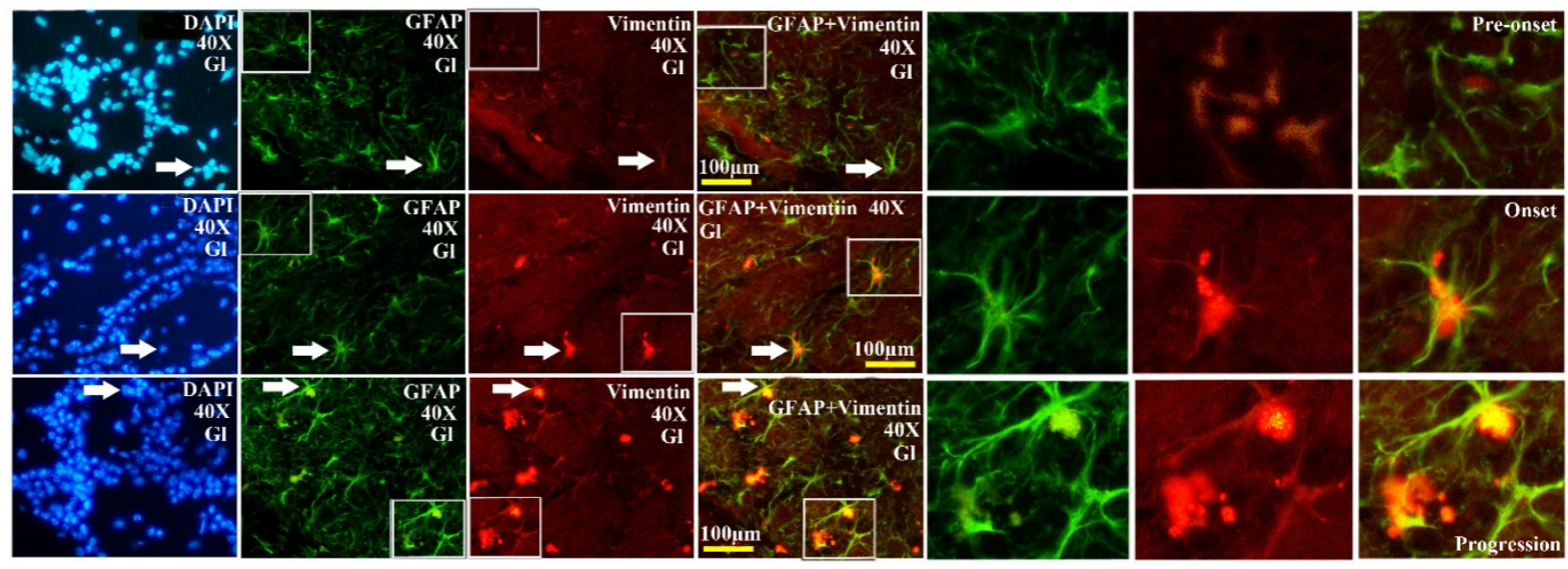

Figure 6. The representative images amplified by 40 times of vimentin positive cells distribution in the $\mathrm{Gl}$ of $\mathrm{OB}$ at the pre-onset, onset and progression stages of the SODI G93A transgenic mice. The amount of vimentin positive cells gradually increased from the pre-onset to onset to progression stages in the SOD1 G93A transgenic mice. The most significant increase stage of vimentin positive cells was at the progression stage. Almost increased vimentin positive cells were the astrocyte cell. 
the $\mathrm{Gl}$ was the most, that in the $\mathrm{AOB}, \mathrm{OB}$ core, GRO, MI and IPL were the secondary, that in the other regions were the fewest (Fig. 1,3). The highest density of VCCs was in the AOB, the secondary was in the OB core, GRO, Gl and MI, the other regions were sparse (Fig. 4). Based on this studied results were highly consistent with our previous studied results [34, 35], we speculated that the vimentin-containing NPCs in the Gl, AOB, OB core, GRO, MI and IPL might be a potential NST yet. Therefore, our study suggested that NST also might extensively distribute in the different regions of $\mathrm{OB}$, and implied that there existed the abundant NST resource in the OB of adult mice, they could differentiate into the astrocyte, oligodendrocyte and neuron cells, were a potential neuroregenerative resource.

\section{The distributed features of VCCs in the adult OB of the SOD1 G93A transgenic mice: a potential astrocyte neuroregenerative response}

In order to further analyzing the distributed features of VCCs in the adult OB of the SOD1 G93A transgenic mice, we compared the difference of VCCs distribution in the different regions of $\mathrm{OB}$ at the different stages between the SOD1 wild-type and G93A transgenic mice. The result revealed that there was the significant difference in the distributed regions, amount, density and neural cells. In the SOD1 G93A transgenic mice, all VCCs significantly increased in the $\mathrm{OB}$ core and the $\mathrm{Gl}$ of adult $\mathrm{OB}$ at the pre-onset, onset and progression stages (Fig. 3, 5, 6). Among them, the most significant increase of VCCs amount was in the $\mathrm{OB}$ core during the pre-onset, onset and progression stages, there weren't the significant increase of VCCs in the other regions of OB at the whole course of ALS-like disease (Fig. 3).

Based on the well-established pathological manifestations of the SOD1 G93A transgenic mice [36-39], we analyzed the distribution of VCCs in the $\mathrm{OB}$ during the pre-onset, onset and progression of the SOD1 G93A transgenic mice. Notably, there were the dynamic changes of VCCs in the OB during the pre-onset, onset and progression stages of the SOD1 G93A mice as compared to the age-matched control mice. The increase of VCCs gradually enhanced in the $\mathrm{OB}$ core and the $\mathrm{Gl}$ of $\mathrm{OB}$ during the pre-onset, onset and progression stages of SOD1 G93A mice. Particularly in the $\mathrm{OB}$ core at the pre-onset, onset and progression stage of ALS (Fig. 3, 5, 6). No decreased VCCs in the entire OB occurred in all stages (Fig. 3). All increased vimentin-containing NPCs were the astrocyte cells (Fig. 5, 6). In view of our previous studied results [35], our data suggested that there were extensively NST proliferation in the OB core and

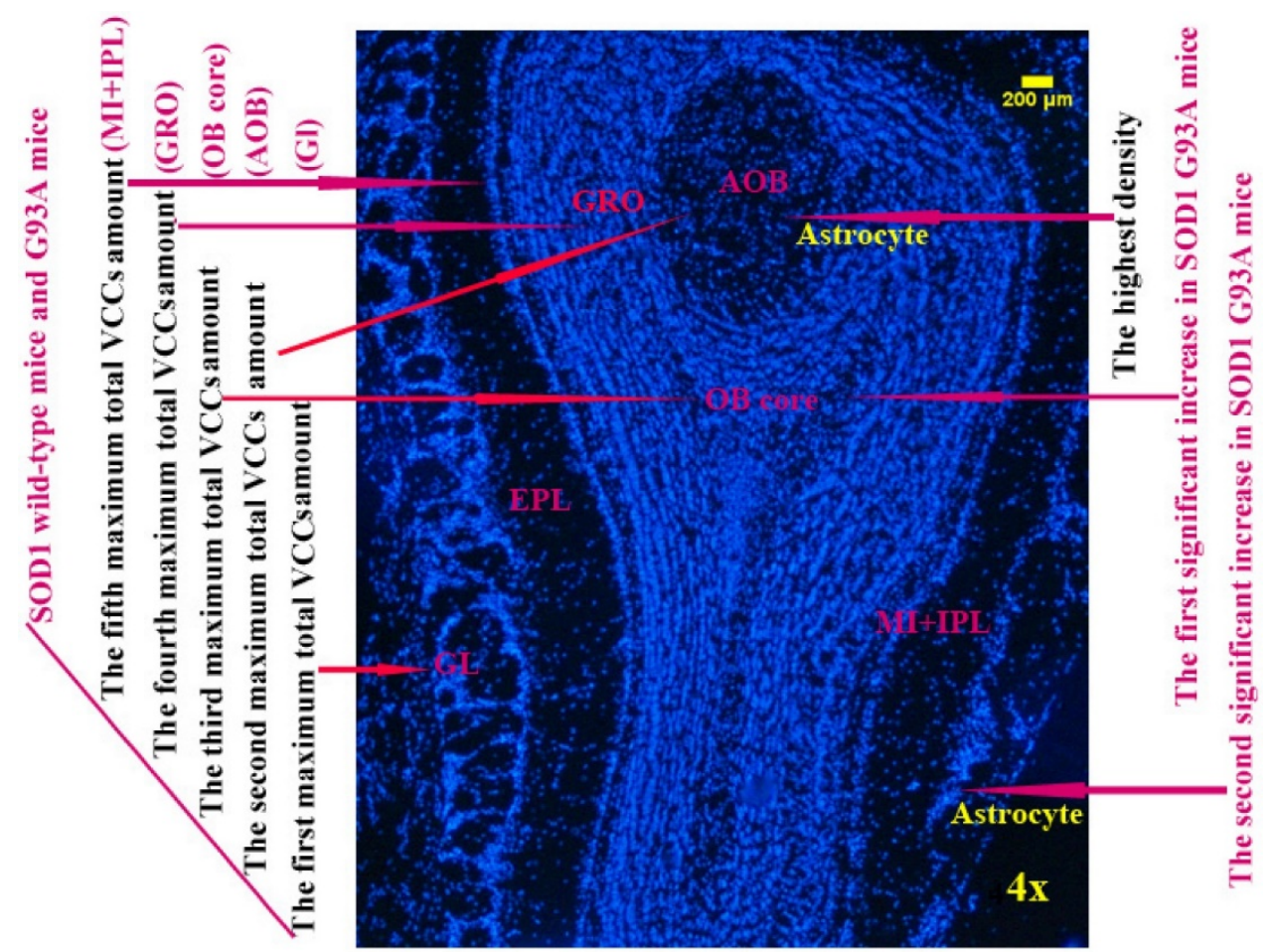

Figure 7. The diagram of the distributed features of VCCs in the OB of the SOD1 wild-type and G93A transgenic mice. The rank order of VCCs amount in the entire $O B$ of SODI wild-type mice was $G \mid>A O B>O B$ core $>G R O>M I+\mid P L>$ the others. The rank order of $V C C s$ density in the entire $O B$ was $A O B>O B$ core $>\mathrm{GRO}>\mathrm{Gl}>\mathrm{Ml}>$ the others in the SODI wild-type mice and G93A transgenic mice. The amount of vimentin positive cells gradually increased from the pre-onset to onset to progression stages in the OB core and GI of the SODI G93A transgenic mice, the most significant increase of vimentin positive cells was in the OB core region at the progression stage, almost all increased vimentin positive cells were the astrocyte cells. 
the $\mathrm{Gl}$ of adult $\mathrm{OB}$ at the pre-onset, onset and progression stages of the ALS-like SOD1 G93A transgenic mice, but NSTs didn't migrate between the different anatomic regions of $\mathrm{OB}$, and all proliferated NST cells might differentiate into the astrocyte cells, which implied that there was a significant neuroregenerative response of the astrocyte cells in the disease course of ALS, which was a potential endogenous neuroregenerative resource at the pathological condition of ALS, and was a potential hopeful way to induce its proliferating, migrating, differentiating into the neural cells, to repair the damaged motor neuron through the cell replacement, the neurotrophic factor secretion, the glutamate transporter regulation, the modulation of neuro-inflammation and others.

To the best of our knowledge, this might be the first systematic report on the organization, distribution, proliferation, differentiation and potential functionality of the vimentin-containing NPCs in the $\mathrm{OB}$ of adult mice during the different disease courses of the ALS-like SOD1 G93A transgenic mice. The alterations of the vimentin-containing NPCs in this study were consistent with our previously reported organization, proliferation, distribution and differentiation patterns of NPCs in the ALS-like SOD1 G93A transgenic mice $[34,35]$. Therefore, our study suggested that there existed an extensively increase of the NSTs, and the neuroregenerative and self-recovered functions in the $\mathrm{OB}$ at the pre-onset, onset and progression stages of the ALS-like SOD1 G93A transgenic mice, which was a hopeful potential resource of neural self-repair in ALS. The inducement of their proliferation, differentiation, migration to the lesion regions is a possible novel treatment way of ALS. Moreover, several transplantation trials of $\mathrm{OB}$ ensheathing cells (OECs) in the ALS patients exhibited some short-term outcome. The OECs implanted in the intracranial key points for neural network restoration, the spinal intraspinal impaired segments and/or the bilateral corona radiata involving the pyramidal tracts of the frontal lobes, which revealed the benefit to the ALS patients including both the bulbar onset and limb onset subtypes, these results indicated that the transplantation of OECs was able to slow the rate of clinical progression of ALS in the first four months of post-transplantation [40-42]. The cells from the OB of normal animal including the human donor can be isolated, cultured, coated by the special medium, transplanted into CNS to cure or prevent the disease of CNS including ALS [43-50]. Therefore, the NPCs of VCCs in the normal OB also are an important resource of exogenous NSTs transplantation, and one of ideal donors of the exogenous transplantation of NSTs.
The past studies reported that the motor neuron degeneration triggered the endogenous NPCs niches in the spinal cord to proliferate, differentiate into the novel neural cells, migrate to the lesion regions of spinal cord, and promoted neuroregeneration in the SOD1 G93A ALS mice model, however, these studied data testified that the limited number of endogenous NPCs was likely insufficient to combat the progressive motor neuron degeneration associated with ALS [10-12, 34]. For that, our study aimed to explorer the distributed feature of NPCs at the pathological conditions of ALS, search the clues for moderating the proliferation, differentiation and migration, improve the repairing power of NPCs at the pathological condition of ALS. The present study will allow us to explore the potential therapeutic pathways stimulating the endogenous astrocyte NPCs to proliferate, differentiate and migrate to the lesion regions, to exert the functional replacement of degenerated neurons, the neurotrophic factor secretion, the glutamate transporter regulation, the modulation of neuro-inflammation and others in the treatment of ALS. Our data provided some evidences that the potential source of the astrocyte stem cells derived from VCCs in the $\mathrm{OB}$ had the capable differentiating into the astrocyte cell, oligodendrocyte cell and potentially even neuron cells, and some useful clue for enhancing the natural endogenous self-repair function through activating the endogenous NPC/NST niches.

In conclusion, our data demonstrated that there was an extensive NPCs/NSTs distribution in the OB of adult mice, these NPCs had the potential of neuroregeneration in the pathological condition of ALS, might be a hopeful treatment way of ALS by improving its potentials of proliferation, differentiation and even inducing its migration to the lesion regions of ALS. The inducing and regulating methods of the endogenous NPCs would avoid a lot of side effects of the exogenous NPCs/NSTs transplantation, was an ideal potential candidate method in the therapy of ALS and the other neurodegenerative disease of CNS. The next important step will be to explore how to induce the endogenous NPCs proliferation, differentiation and migration to the lesion regions of ALS, to repair the degenerative motor neurons of ALS through the cell replacement, the neurotrophic factor secretion, the glutamate transporter regulation, the modulation of neuro-inflammation and others.

\section{Abbreviations}

ALS, amyotrophic lateral sclerosis; VCCs, vimentin-containing cells; GFAP, glial fibrillary acidic protein; NeuN, hexaribonucleotide Binding 
Protein-3; Oligo-2, anti-oligodendrocyte antibody-2; DAPI, 4',6-diamidino-2-phenylindole; OB, olfactory bulb; Gl, glomerular layer; AOB, accessory OB; GRO, granular cell layer; MI, mitral cell layer; IPL, internal plexiform layer.

\section{Acknowledgements}

This work was supported by the National Natural Science Foundation of China (30560042, $81160161,81360198)$, the education department of Jiangxi province (GJJ10303) and Jiangxi provincial department of science and technology ([2014]-47, 20142BBG70062) for funding this study.

\section{Authors' contributions}

All authors contributed significantly to this research and preparation of the manuscript. R.X. and C.T. conceived and designed the experiments and wrote the manuscript. C.T., L.Z., W.G., H.L., J.L., J.Z., X.Z. and Y.L. performed the experiments and analyzed the data. C.T., L.Z., W.G., H.L., J.L., J.Z. and $X . Z$. are the first author and contributed equally to the work. All authors have been involved in the drafting, critical revision and final approval of the manuscript for publication. All authors agree to be accountable for all aspects of the work in ensuring that questions related to the accuracy or integrity of any part of the work are appropriately investigated and resolved.

\section{Competing Interests}

The authors have declared that no competing interest exists.

\section{References}

1. Nelson LM. Epidemiology of ALS. Clinical neuroscience. 1995; 3: 327-31.

2. Cleveland DW, Rothstein JD. From Charcot to Lou Gehrig: deciphering selective motor neuron death in ALS. Nature reviews neuroscience. 2001; 2: 806-19.

3. Mitchell JD, Borasio GD. Amyotrophic lateral sclerosis. Lancet. 2007; 369: 2031-41.

4. Wijesekera LC, Leigh PN. Amyotrophic lateral sclerosis. Orphanet journal of rare diseases. 2009; 4: 3.

5. Salameh JS, Brown RH Jr, Berry JD. Amyotrophic Lateral Sclerosis: Review. Seminars in neurology. 2015; 35:469-76.

6. Blasco H, Mavel S, Corcia P, Gordon PH. The glutamate hypothesis in ALS: pathophysiology and drug development. Current medicinal chemistry. 2014; 21: $3551-75$

7. Miller RG, Mitchell JD, Moore DH. Riluzole for amyotrophic lateral sclerosis (ALS)/motor neuron disease (MND). Cochrane database of systematic reviews. 2012; 3: CD001447.

8. Cheah BC, Vucic S, Krishnan AV, Kiernan MC. Riluzole, neuroprotection and amyotrophic lateral sclerosis. Current medicinal chemistry. 2010; 17: 1942-199.

9. Contestabile A. Amyotrophic lateral sclerosis: from research to therapeutic attempts and therapeutic perspectives. Current medicinal chemistry. 2011; 18 : 5655-65.

10. Chi L, Ke Y, Luo C, Li B, Gozal D, Kalyanaraman B, et al. Motor neuron degeneration promotes neural progenitor cell proliferation, migration, and neurogenesis in the spinal cords of amyotrophic lateral sclerosis mice. Stem cells. 2006: $24 \cdot 34-3$

11. Chi L, Gan L, Luo C, Lien L, Liu R. Temporal response of neural progenitor cells to disease onset and progression in amyotrophic lateral sclerosis-like transgenic mice. Stem cells and development. 2007; 16: 579-88

12. Lindvall $\mathrm{O}, \mathrm{Kok}$ aia $\mathrm{Z}$. Stem cells in human neurodegenerative disorders--time for clinical translation? Journal of clinical investigation. 2010; 120: 29-40.
13. Traub R, Mitsumoto $H$, Rowland LP. Research advances in amyotrophic lateral sclerosis, 2009 to 2010 . Current neurology and neuroscience reports. 2011; 11: 67-77.

14. Haidet-Phillips AM, Maragakis NJ. Neural and glial progenitor transplantation as a neuroprotective strategy for Amyotrophic Lateral Sclerosis (ALS). Brain research. 2015; 1628: 343-50.

15. Bucchia M, Ramirez A, Parente V, Simone C, Nizzardo M, Magri F, et al. Therapeutic development in amyotrophic lateral sclerosis. Clinical therapeutics. 2015; 37: 668-80.

16. Thomsen GM, Gowing G, Svendsen S, Svendsen CN. The past, present and future of stem cell clinical trials for ALS. Experimental neurology. 2014; 262(Pt B): 127-37.

17. Silani V, Calzarossa C, Cova L, Ticozzi N. Stem cells in amyotrophic lateral sclerosis: motor neuron protection or replacement? CNS Neurol Disord Drug Targets. 2010; 9: 314-24

18. Peters OM, Ghasemi M, Brown RH Jr. Emerging mechanisms of molecular pathology in ALS. Journal of clinical investigation. 2015; 125: 1767-79.

19. Brites D, Vaz AR. Microglia centered pathogenesis in ALS: insights in cell interconnectivity. Frontiers in Cellular Neuroscience. 2014; 8: 117.

20. Phatnani $\mathrm{H}$, Maniatis T. Astrocytes in neurodegenerative disease. Cold Spring Harbor perspectives in biology. 2015; 7pii: a020628.

21. Haidet-Phillips AM, Maragakis NJ. Neural and glial progenitor transplantation as a neuroprotective strategy for Amyotrophic Lateral Sclerosis (ALS). Brain research. 2015; 1628(Pt B): 343-50.

22. Nicaise C, Mitrecic D, Falnikar A, Lepore AC. Transplantation of stem cell-derived astrocytes for the treatment of amyotrophic lateral sclerosis and spinal cord injury. World journal of stem cells. 2015; 7: 380-98.

23 Cao QL, Zhang YP, Howard RM, Walters WM, Tsoulfas P, Whittemore SR. Pluripotent stem cells engrafted into the normal or lesioned adult rat spinal cord are restricted to a glial lineage. Experimental neurology. 2001; 167: 48-58.

24. Tanna T, Sachan V. Mesenchymal stem cells: potential in treatment of neurodegenerative diseases. Current stem cell research \& therapy. 2014; 9: 513-21.

25. Allers C, Jones JA, Lasala GP, Minguell JJ. Mesenchymal stem cell therapy for the treatment of amyotrophic lateral sclerosis: signals for hope? Regenerative Medicine. 2014; 9: 637-47.

26. Chen KS, Sakowski SA, Feldman EL. Intraspinal stem cell transplantation for amyotrophic lateral sclerosis. Annals of neurology. 2016; 79: 342-53.

27. Coatti GC, Beccari MS, Olávio TR, Mitne-Neto M, Okamoto OK, Zatz M. Stem cells for amyotrophic lateral sclerosis modeling and therapy: myth or fact? Cytometry A. 2015; 87: 197-211.

28. Mariano ED, Batista CM, Barbosa BJ, Marie SK, Teixeira MJ, Morgalla M, et al. Current perspectives in stem cell therapy for spinal cord repair in humans: a review of work from the past 10 years. Arquivos de Neuro-Psiquiatria. 2014; 72: 451-6.

29. Lunn JS, Sakowski SA, Feldman EL. Concise review: Stem cell therapies for amyotrophic lateral sclerosis: recent advances and prospects for the future. Stem Cells. 2014; 32: 1099- 109.

30. Meamar R, Nasr-Esfahani MH, Mousavi SA, Basiri K. Stem cell therapy in amyotrophic lateral sclerosis. Journal of clinical neuroscience. 2013; 20: 1659-63.

31. Faravelli I, Riboldi G, Nizzardo M, Simone C, Zanetta C, Bresolin N, Comi GP, Corti S. Stem cell transplantation for amyotrophic lateral sclerosis: therapeutic potential and perspectives on clinical translation. Cellular and molecular life sciences. 2014; 71: 3257-68.

32. Silani V, Corbo M. Cell-replacement therapy with stem cells in neurodegenerative diseases. Current neurovascular research. 2004; 1: 283-9.

33. Lepore AC, O'Donnell J, Kim AS, Williams T, Tuteja A, Rao MS, Kelley LL, Campanelli JT, Maragakis NJ. Human glial-restricted progenitor transplantation into cervical spinal cord of the SOD1 mouse model of ALS. PLoS One. 2011; 6: e25968.

34. Zhou $Y, L u ~ Y$, Fang $X$, Zhang J, Li J, Li S, et al. An astrocyte regenerative response from vimentin-containing cells in the spinal cord of amyotrophic lateral sclerosis's disease-like transgenic (G93A SOD1) mice. Neurodegenerative diseases. 2015; 15: 1-12.

35. Xu R, Wu C, Tao Y, Yi J, Yang Y, Yang R, et al. Description of distributed features of the nestin-containing cells in brains of adult mice: a potential source of neural precursor cells. Journal of neuroscience research. 2010; 88: 945-56.

36. Rosen DR, Siddique T, Patterson D, Figlewicz DA, Sapp P, Hentati A, et al. et al. Mutations in $\mathrm{Cu} / \mathrm{Zn}$ superoxide dismutase gene are associated with familial amyotrophic lateral sclerosis. Nature. 1993; 362: 59-62.

37. Gurney ME, Pu H, Chiu AY, Dal Canto MC, Polchow CY, Alexander DD, et al. Motor neuron degeneration in mice that express a human $\mathrm{Cu}, \mathrm{Zn}$ superoxide dismutase mutation. Science. 1994; 264: 1772-5.

38. Henriques A, Pitzer C, Schneider A. Characterization of a novel SOD-1(G93A) transgenic mouse line with very decelerated disease development. PLoS one. 2010; 5: e15445.

39. Gurney ME. The use of transgenic mouse models of amyotrophic lateral sclerosis in preclinical drug studies. Journal of the neurological sciences. 1997; 152 Suppl 1: S67-73.

40. Huang $\mathrm{H}$, Tan $\mathrm{K}$, Chen $\mathrm{L}$, Xue $\mathrm{Y}$, Wang $\mathrm{H}$, Xi H, et al. MR spectroscopy evaluation and short-term outcome of olfactory ensheathing cells transplantation in amyotrophic lateral sclerosis patients. Zhongguo Xiu Fu Chong Jian Wai Ke Za Zhi. 2007; 21: 52-7. 
41. Chen L, Chen D, Xi H, Wang Q, Liu Y, Zhang F, et al. Olfactory ensheathing cell neurorestorotherapy for amyotrophic lateral sclerosis patients: benefits from multiple transplantations. Cell transplantation: The regenerative medicine journal. 2012; 21 Suppl 1: S65-77.

42. Huang H, Chen L, Xi H, Wang H, Zhang J, Zhang F, et al. Fetal olfactory ensheathing cells transplantation in amyotrophic lateral sclerosis patients: a controlled pilot study. Clinical transplantation. 2008; 22: 710-8.

43. Marei HE, Lashen S, Farag A, Althani A, Afifi N, A AE, et al. Human olfactory bulb neural stem cells mitigate movement disorders in a rat model of Parkinson's disease. Journal of cellular physiology. 2015; 230: 1614-29.

44. Tello Velasquez J, Watts ME, Todorovic M, Nazareth L, Pastrana E, Diaz-Nido $\mathrm{J}$, et al. Low-dose curcumin stimulates proliferation, migration and phagocytic activity of olfactory ensheathing cells. PLoS One. 2014; 9: e111787.

45. Honoré A, Le Corre S, Derambure C, Normand R, Duclos C, Boyer O, et al. Isolation, characterization, and genetic profiling of subpopulations of olfactory ensheathing cells from the olfactory bulb. Glia. 2012; 60: 404-13.

46. Graziadei PP. Olfactory organ culture in vivo and in vitro. Cytotechnology. 1993; 11: 3-6.

47. Higginson JR, Barnett SC. The culture of olfactory ensheathing cells (OECs)--a distinct glial cell type. Experimental neurology. 2011; 229: 2-9.

48. Wewetzer K, Verdú E, Angelov DN, Navarro X. Olfactory ensheathing glia and Schwann cells: two of a kind? Cell and tissue research. 2002; 309: 337-45.

49. Mendoza AS. The cell coat of the developing olfactory epithelium in the chick. Cell and tissue research. 1980; 207: 227-32

50. Mendoza AS, Breipohl W. The cell coat of the olfactory epithelium proper and vomeronasal neuroepithelium of the rat as revealed by means of the Ruthenium-red reaction. Cell and tissue research. 1983; 230:139-46. 